\title{
Toxicité du carbofuran et activité synergique de l'atrazine sur son action vis-à-vis de quelques espèces animales
}

\author{
P.-A. Lardier ${ }^{1}$ et M. Schiavon ${ }^{2}$ \\ 1 UNCAA, 83/85, avenue de la Grande-Armée, 75782 Paris; \\ 2 ENSAIA, 2, avenue de la Forêt-de-Haye, 54500 Vandceuvre, France
}

(reçu le 17 octobre 1988, accepté le 13 mars 1989)

\begin{abstract}
Résumé - L'utilisation simultanée de molécules phytosanitaires conduit à des changements de l'activité attendue. La synergie carbofuran-atrazine se traduit par une plus grande toxicité du mélange vis-à-vis des organismes testés que ne le laisserait supposer la somme des effets des produits, utilisés séparément. Les collemboles, les vers de terre, les grillons et les carabiques ont fait l'objet de divers tests biologiques (test du film sec, application topique ou test par immersion), dans lesquels les $\mathrm{CL}_{50}$ (ou $\mathrm{DL}_{50}$ ) d'un standard carbofuran étaient comparées au mélange carbofuran-atrazine. Les bonnes pratiques agricoles nécessitent une bonne connaissance des produits phytosanitaires : la compatibilité biologique devrait être étudiée pour éviter les erreurs.
\end{abstract}

carbofuran - atrazine - synergie - test biologique

Summary - Toxicity of carbofuran and synergy with atrazine in insects and worms. Mixed use of both carbofuran and atrazine is more toxic in many organisms than it will be expected when applied separately. Springtails, earthworms, grasshoppers and ground-beetles have been tested in various biological tests (dry film test, topic application or immersion test) in which were compared $C L_{50}$ (or $D L_{50}$ ) of the standard carbofuran and the mixture carbofuran-atrazine. Good agricultural practice requires a good knowledge of the phytosanitary products : biological compatibility must be studied to avoid mistakes.

carbofuran - atrazine - synergy - biological tests

\section{Introduction}

Dans l'agriculture moderne, de nombreux produits phytosanitaires sont utilisés pour protéger les cultures contre leurs ennemis. Ces produits sont appliqués soit successivement, soit simultanément afin de réduire le temps de travail et donc le coût des traitements.

Ainsi, certains produits se trouvent en même temps dans le milieu et cette présence simultanée peut entraîner des interactions et modifier leur activité de façon inattendue. En dehors de l'absence d'interaction, les travaux déjà réalisés montrent des possibilités de renforcement d'activité : synergie (Sun et Johnson, 1972; Ozaki, 1983; Dai et Sun, 1984) ou de perte d'activité : antagonisme (Bliss, 1939; Liu et al., 1982; Wilde et al., 1984).

Cette augmentation ou inhibition de l'activité biologique peut conduire à certains désagréments ou accidents, affectant la culture, la faune ou la flore auxiliaire : contrôle insuffisant du parasite visé, destruction d'insectes utiles... A moyen terme, ces effets peuvent retentir sur l'environnement au sens large. II nous a donc paru intéressant d'examiner l'interaction de 2 molécules souvent utilisées en même temps en culture de maïs : l'atrazine et le carbofuran. L'activité insecticide du carbofuran a été observée par divers tests biologiques sur collemboles, vers de terre, carabes et grillons, en présence ou en l'absence d'herbicide. 


\section{Matériel et Méthodes}

Seuls les tests biologiques permettent de mesurer l'activité biocide d'un produit. Pour confirmer l'effet de l'interaction carbofuran-atrazine, notre étude a fait appel à 3 méthodes de tests.

\section{Test sur collemboles : Folsomia candida Willem}

La technique proposée par Sun (1957) est simple et nous l'avons adaptée à nos besoins. Dans une boîte de Petri de $4 \mathrm{~cm}$ de diamètre, garnie d'une rondelle de papier Chardin, on dépose : soit $1 \mathrm{ml}$ de solution insecticide de concentration connue (modalité standard), soit $1 \mathrm{ml}$ de solution insecticide et herbicide, également de concentrations connues.

Le contenu des boîtes est ensuite évaporé à sec. Après évaporation totale, le papier est réhumidifié par $0,3 \mathrm{ml}$ d'eau, puis on dépose dans chaque boîte 10 collemboles adultes provenant de notre élevage (Lardier, 1987). Les boîtes de Petri sont alors refermées et placées à température contrôlée $\left(20 \pm 1^{\circ} \mathrm{C}\right)$ pendant $24 \mathrm{~h}$, à l'obscurité, dans une atmosphère saturée en eau. Après ce laps de temps, il est procédé au dénombrement des survivants.

Les matières actives sont apportées dans du méthanol. Les concentrations utilisées pour le carbofuran vont de 0 à $0,50 \mathrm{mg} . \mathrm{l}^{-1}$. Pour l'atrazine, après essais préliminaires, la concentration a été fixée à 0 ou 2 mg.l-1.

\section{Test sur vers de terre : Eisenia fetida Savigny}

Ce test comparatif rapide consiste en une immersion des vers de terre dans des solutions insecticides et/ou herbicides, de concentrations variables, pendant $3 \mathrm{~h}$. II simule l'engorgement des galeries des vers à la suite de précipitations importantes, sous condition de plein champ.

Bien que rarement rencontré dans les champs, nous avons retenu pour notre expérimentation Eisenia fetida, facile à obtenir en grand nombre. Trois lots ont été constitués suivant la provenance des vers.

Le premier lot a été prélevé le jour même de l'expérimentation dans le tas de fumier de la ferme expérimentale de I'ENSAIA Nancy. Les 2 autres lots proviennent d'élevages : élevage de I'IRCHA* à Vert-le-Petit, où les vers sont élevés sur terreau et paille : élevage de l'INRA à Dijon, où ils sont élevés sur ordures ménagères.

Les vers testés sont tous des adultes d'un poids compris entre 300 et $500 \mathrm{mg}$. Après leur immersion de $3 \mathrm{~h}$, par groupe de 15, dans des piluliers contenant $100 \mathrm{ml}$ de solution aqueuse de pesticides à $20^{\circ} \mathrm{C}$, ils sont transférés dans des boîtes de Petri, de $14 \mathrm{~cm}$ de diamètre, contenant du terreau humide à $20^{\circ} \mathrm{C}$, et placés à l'obscurité pendant $24 \mathrm{~h}$. La mortalité est alors estimée.

\section{Test sur carabes : Poecilus cupreus Linné}

Les insectes testés sont des carabiques adultes, récoltés au champ grâce à des pièges de Barber relevés quotidiennement. Le choix de l'espèce a été fait sur la base d'abondance relative dans les piégeages. Le test

* IRCHA : Institut de Recherche de Chimie Appliquée (Vert-lePetit-91). consiste en un dépôt d'une gouttelette $(2 \mu \mathrm{l})$ de solution acétonique d'insecticide (ou d'insecticide-herbicide) à la limite céphalothoracique (face ventrale), grâce à un microapplicateur d'Arnold, sur des animaux préalablement endormis au $\mathrm{CO}_{2}$. Les animaux sont ensuite placés par lots de 15 (ayant reçu le même traitement) dans des récipients contenant de l'eau et de la nourriture pour $24 \mathrm{~h}$, à une température de $20^{\circ} \mathrm{C}$. Les survivants sont ensuite dénombrés.

\section{Test sur grillons : Grillus domesticus Linné}

Les insectes utilisés proviennent de notre élevage. Dans le cas des adultes, nous avons procédé de la même manière que pour les carabes. Pour les différents stades larvaires $L_{1}, L_{2}, L_{3}, L_{4-5}$, les tests ont été menés à l'aide de la technique du film sec.

Stade $L_{1}$ Dix oeufs de grillons sont déposés dans différentes boîtes de Petri garnies de papier Chardin, humidifié par $1 \mathrm{ml}$ d'une solution aqueuse d'insecticide (ou insecticide-herbicide) de concentration définie.

Stades $L_{2}, L_{3}, L_{4-5}$. Après engourdissement par le froid, 10 larves sont placées dans différentes boites de Petri de $10 \mathrm{~cm}$ de diamètre garnies de papier Chardin, humidifié par $3 \mathrm{ml}$ de solution aqueuse d'insecticide (ou d'insecticide-herbicide) de concentration définie.

Dans tous les cas, les tests sont effectués à $20 \pm$ $1^{\circ} \mathrm{C}$ et la mortalité est évaluée après $24 \mathrm{~h}$.

\section{Les produits}

Dans toutes les expériences réalisées, les solutions testées sont préparées à partir des produits techniques (test collemboles) ou des produits commerciaux : Curater et Gesaprime 50 (test sur vers, carabidés et grillidés).

Après différents essais, les concentrations de carbofuran retenues ont été les suivantes :

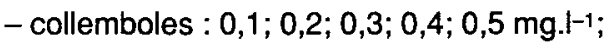

- vers de terre : $0 ; 0,5 ; 1 ; 2 ; 4 ; 8 \mathrm{mg} . \mathrm{I}^{-1}$;

- carabes : $0 ; 2,5 ; 5 ; 10 \mathrm{mg.t} .^{-1}$;

- grillons adultes : $0 ; 25 ; 50 ; 100 ; 150$ mg.l-1.

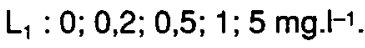

$L_{2}, L_{3}, L_{4-5},: 0 ; 1 ; 2 ; 5 ; 10$ mg..$^{-1}$.

Pour l'atrazine, des expériences annexes ont permis d'établir sa non-toxicité pour des concentrations inférieures à 2, 4 et $10 \mathrm{mg} \mathrm{l}^{-1}$, respectivement, sur vers de terre, carabiques et collemboles. Une concentration de $2 \mathrm{mg}^{-1}$ a été retenue pour l'ensemble des essais.

\section{Traitement des résultats}

Notre standard est constitué par la modalité "Carbofuran seul", qui est incluse dans toutes les expériences et nous permet de vérifier la régularité du matériel biologique dans sa sensibilité au carbofuran. Toute variation du standard supérieure à $10 \%\left(\mathrm{CL}_{50} \text { ou } \mathrm{DL}_{50}\right)^{\star}$ entraîne l'annulation de l'essai dont il est la référence. Toutes les données recueillies pour chaque test sont

\footnotetext{
- $\mathrm{CL}_{50}$ : concentration létale centrale ou concentration de la substance toxique qui provoque la mort de $50 \%$ des organismes exposés.

$\mathrm{DL}_{50}$ : dose létale centrale ou quantité de substance toxique qui provoque la mort de $50 \%$ des organismes auxquels elle a été appliquée.
} 
traitées en régression linéaire, suivant la méthode des moindres carrés pondérés, introduite par Bliss (1935) et développée par Finney (1964), afin de calculer les $\mathrm{CL}_{50}$ (dans le cas des tests par film sec ou immersion) ou les $\mathrm{DL}_{50}$ (dans le cas des tests par application topique).

La linéarité des droites et l'identité des modes d'action des traitements sont vérifiés par un $\chi^{2}$, tandis qu'un $t$ de Student permet de vérifier la significativité des résultats obtenus.

\section{Résultats et discussion}

Les résultats présentés dans le Tableau I montrent clairement que l'interaction carbofuran-atra- zine se traduit systématiquement par un renforcement de l'activité insecticide du carbofuran, obtenu par combinaison avec des solutions d'atrazine non toxiques pour les organismes. Cet effet synergique se manifeste quelles que soient l'espèce étudiée ou la technique utilisée; il n'est cependant pas constant. Selon les organismes soumis à l'expérience, le coefficient de synergie varie de 1,1 à 2,4 . De plus, à côté de ces différences interspécifiques, les tests montrent une variabilité intraspécifique dans la sensibilité aux xénobiotiques.

Les collemboles ne sont pas des organismes habituellement visés par le carbofuran (ni par

Tableau l. Synergie observée sur divers organismes par différentes méthodes (durée des tests : 24 h).

\begin{tabular}{|c|c|c|c|}
\hline Organisme, origine & Technique & Matières actives & $\begin{array}{l}C L_{50} \\
(m g / l)\end{array}$ \\
\hline
\end{tabular}

Collemboles adultes

(élevage laboratoire)

adultes (champ)

\section{ENSAIA \\ (fumier)}

IRCHA

(élevage)

INRA

(élevage)

Immersion

Immersion

Immersion

CF-At

CF

CF-At

CF

CF-At

CF

CF-At

$0,31 \pm 0,03$

$0,18 \pm 0,02$

$2,2 \pm 0,7$

$0,9 \pm 0,2$

$6,36 \pm 0,95$

$4,51 \pm 0,43$

$30,26 \pm 2,34$

$15,38 \pm 1,21$

* Application
topique

CF

CF-At

$12,96 \pm 4,0$

$6,28 \pm 1,6$

Larves $L_{1}$

Film

CF

CF-At

$2,59 \pm 0,22$

$1,27 \pm 0,17$

Larves $L_{2}$

Film

CF

CF-At

$5,51 \pm 0,14$

$4,00 \pm 0,05$

$7,51 \pm 0,20$

$6,59 \pm 0,57$

CF-At

CF

CF-At

$17,47 \pm 2,61$

$12,22 \pm 2,17$

CF

CF-At

$618 \pm 28$

$417 \pm 17$
1,72 
l'atrazine); ils sont cependant des modèles très précieux pour suivre avec précision les interactions, car très sensibles aux substances susceptibles de les intoxiquer. L'atrazine seule n'est pas toxique pour les collemboles aux concentrations comprises entre 0 et $10 \mathrm{mg} . \mathrm{l}^{-1}$. Elle entraîne cependant une synergie significative et reproductible pour des concentrations de l'ordre de 1 à 2 mg.t-1. En dessous de cette concentration, le mélange carbofuran-atrazine conduit à des mortalités plus fortes que le carbofuran seul, mais la variabilité est très grande d'une manipulation à la suivante. Au-delà de $2 \mathrm{mg}^{-1}{ }^{-1}$, on n'observe pas d'accroissement de la toxicité du mélange à mesure que la part relative de l'atrazine devient plus forte (Lardier, 1987). La valeur retenue (2 mg.l-1) est donc nécessaire mais suffisante pour permettre l'observation de l'effet interactif de façon fiable. Elle est, de plus, pertinente en regard des concentrations observées dans les champs après traitements. L'activité synergique de l'atrazine sur la toxicité du carbofuran est donc bien établie sur collemboles, particulièrement pour des concentrations proches de $2 \mathrm{mg} . \mathrm{l}^{-1}$.

Chez les vers, la sensibilité aux pesticides est différente selon les espèces. Lumbricus herculeus est plus sensible qu'Eisenia fetida (Gilman et Vardanis, 1974; Heimbach, 1985). Nos test sur Eisenia fetida permettent d'observer une variabilité intraspécifique dans la toxicité, mais aussi dans la synergie. $\mathrm{La} \mathrm{CL}_{50}$ varie de 2,2 à $30,26 \mathrm{mg} . \mathrm{l}^{-1}$ en absence d'atrazine et de 0,9 à 15,38 mg.l-1 en présence d'atrazine (le coefficient de synergie varie de 1,41 à 2,44 ).

Ces différences observées dans les $C_{50}$ chez cette espèce semblent liées aux milieux sur lesquels les vers ont été élevés. Les vers d'élevage sont plus tolérants que les vers "sauvages" et cela est d'autant plus marqué que le milieu d'élevage est, a priori, hostile, voire agressif, par rapport au fumier. Toutefois, on ne note pas de relation entre la sensibilité des vers exprimée par la $\mathrm{CL}_{50}$ et l'effet synergique.

Les grillons se distinguent des carabes par une moindre sensibilité au carbofuran. Cette différence de sensibilité peut être considérée (chez les adultes) comme une variation interspécifique, et non pas comme une conséquence de la différence de poids entre les individus des 2 espèces. En effet, si l'on prend en compte le poids, ramené au "gramme d'insecte", le carbofuran et le mélange carbofuran-atrazine sont respectivement 3,16 et 3,11 fois plus toxique pour les carabes que pour les grillons. Toutefois, nous noterons que, contrairement aux carabes, les grillons testés sont des animaux d'élevage au laboratoire, ce qui peut indirectement influer sur leur sensibilité aux produits toxiques (absence de stress dû à l'encagement et au changement d'environnement...).

Enfin, si une variation importante de sensibilité au carbofuran des grillons est observée en fonction du stade de l'insecte, en revanche, l'effet synergique carbofuran-atrazine reste sensiblement constant, avec un facteur multiplicatif de 1,4 , excepté pour le stade $L_{1}$ où il atteint la valeur 2 .

\section{Conclusions}

La sensibilité des organismes testés varie selon les espèces, mais aussi à l'intérieur de la même espèce, selon l'âge, le stade et/ou les caractéristiques du milieu de vie. Dans tous les cas testés, le carbofuran et l'atrazine ont interagi et provoqué des effets différents de ceux correspondant à la somme de leurs effets respectifs. Cette synergie n'est pas exceptionnelle; d'autres carbamates peuvent réagir avec l'atrazine (Lardier, 1982) et d'autres triazines avec le carbofuran.

La campagne agricole 1985-1986 nous a révélé des problèmes dus à l'emploi d'un fongicide et d'un insecticide ayant tous deux le label abeille. Dans quelques cas, leur emploi en mélange s'est révélé toxique pour ces Hyménoptères (Anonyme, 1988). Un complément d'étude serait donc intéressant pour bien connaître les effets interactifs des produits phytosanitaires et pour mieux les utiliser, en respectant leurs propriétés intrinsèques mais aussi l'ensemble des éléments de l'agrosystème.

\section{Remerciements}

Les auteurs remercient le PIREN pour le soutien apporté à ces recherches.

\section{Références}

Anonyme (1988) Etude de la toxicité à court terme de mélanges d'insecticide et de fongicide. Rev. Franç. Apic. 477, 383-386

Bliss C.I. (1935) The calculation of dosage mortality curve. Ann. Appl. Biol. 22, 135-167

Bliss C.I. (1939) Mixtures of poisons. Ann. Appl. Biol. 26, 585-592

Dai S.M. \& Sun C.N. (1984) Pyrethroid resistance and synergism in Nilaparvata lugens Stal (Homoptera : Delphacidae) in Taïwan. J. Econ. Entomol. 77, 891897

Finney D.J. (1964) Probit Analysis (2nd ed.). Cambridge University Press, Cambridge

Gilman A.P. \& Vardanis A. (1974) Carbofuran. Comparative toxicity and metabolism in the worms Lumbricus terrestris L. and Eisenia foetida S. J. Agric. Food Chem. 22, 625-628 
Heimbach F. (1985) Comparison of laboratory methods, using Eisenia foetida and Lumbricus terrestris for the assessment of the hazard of chemicals to earthworms. Z. Pflanzenkr. Pflanzenschutz 92, 186193

Lardier P.A. (1982) Contribution à l'étude de la toxicité du thiofanox (Dacamox ${ }^{\circledR}$ ) sur quelques éléments de la faune utile du sol. DEA INPL

Lardier P.A. (1987) De la synergie entre insecticide et herbicide : cas du carbofuran et de l'atrazine. Doctorat INPL

Liu M.Y., Sun C.N. \& Huang S.W. (1982) Absence of synergism of DDT by piperonyl butoxide and DMC in larvae of the Diamondback Moth (Lepidoptera : Yponomentidae). J. Econ. Entomol. 75, 964-965
Ozaki K. (1983) Suppresion of resistance through synergistic combinations with emphasis on planthoppers and leafhoppers infesting rice in Japan. In: Pest Resistance to Pesticides (Georghiou G.P. \& Saito T., eds.). Plenum Press, pp. 595-613

Sun Y.P. (1957) Bioassay of pesticide residues. Adv. Pest Control. Res. 1, 449-496

Sun Y.P. \& Johson E.R. (1972) Quasi-synergism and penetration of insecticides. J. Econ. Entomol. 65, 349353

Wilde G., Kadoum A. \& Mize T. (1984) Absence of synergism with insecticide combinations used on chinch bugs (Heterophera : Lygaeidae). J. Econ. Entomol. 77, 1297-1298 